\title{
Peculiarities of phonon spectra and lattice heat capacity in Ir and Rh.
}

\author{
By M. I. Katsnelson ${ }^{\dagger}$, I. I. Naumov $\ddagger$, A. V. Trefilov, M. N. \\ KhLOPKIN AND K. YU. Khromov \\ Russian Research Centre "Kurchatov Institute", Moscow 123182, Russia \\ †Institute of Metal Physics, Ekaterinburg 620219 ,Russia \\ $\ddagger$ Institue of Physics of Strength and Material Science, Tomsk 644055, Russia
}

\begin{abstract}
A simple pseudopotential model is proposed, which allows the phonon spectra and temperature dependence of the lattice heat capacity of Ir and Rh be described with a high enough accuracy. A careful comparison of the calculated and experimental values of the lattice heat capacity is carried out, with the procedure of the identification of the phonon contribution to the heat capacity and determination of the characteristics (momenta) of the phonon density of states from the experimental values of the total heat capacity of metal at a constant pressure being described in detail. The results of the theoretical calculations explain, in particular, such peculiar feature of Ir and Rh, unusual for cubic metals, as a sharp (more than by a factor of 1.5) decrease in the effective Debye temperature with increasing termperature. The temperature dependence of the mean square amplitude of atomic displacements in Ir and $\mathrm{Rh}$ has been calculated. Basing on the band calculations the manifestation of the Kohn singularities in the phonon spectra of Ir are discussed.
\end{abstract}

\section{$\S 1$. INTRODUCTION}

From the physical point of view iridium and its analogue rhodium stand out by their unusual mechanical properties among other FCC metals (see discussion in Gornostyrev et al 1994). The peculiarities of mechanical properties (at least those inherent to high pure single crystals) should be eventually determined by the specific features of interatomic interactions in metal. Greenberg et al 1990 and Ivanov et al 1994 succeded in constructing of a pseudopotential model of pair forces for these metals wich describes surprisingly well (for transition metals) their elastic properties. In terms of this model the peculiarities of the Ir and Rh defect structure (Ivanov et al 1994, Gornostyrev et al 1994) could be analyzed, and the qualitative explanation of some of their peculiar mechanical properties, primarily, the possibility of brittle failure of single crystals after a long-term stage of plastic deformation could be given. At the same time it was found that in the description of the dispersion curves of phonons in Ir measured by Ivanov et al 1994 the model does not prove to be accurate enough for oscillation modes near the Brillouin zone boundaries. According to the calculations of Ivanov et al 1994 the maximum frequency of the phonon spectrum appears to be overestimated by $25 \%$ comparing with the experimental value found from the results of tunnel experiments (Kraynukov et al 1989), and flattenings in the 
$\langle 110\rangle,\langle 100\rangle$ directions are observed, which are not described by the proposed model.

The main purpose of the present paper is to ascertain the physical nature of these descrepancies, improve the model correspondingly, and calculate a number of thermodynamical properties with the allowance for the corrected phonon spectra.

It may seem that the use of a simple pseudopotential model for these purposes does not correspond to the current state of the quantitative theory of metals, when completely $a b$ initio methods for calculation of phonon spectra have been developed (see, e. g., review Maksimov and Savrasov 1995). However, the description of a wide range of lattice properites, including temperature dependence of different values is still beyond the capabilities of completely $a b$ initio approaches. Moreover if it is taken into account that the pseudopotential approach also gives the pairwise potentials which can be used for the simulation of defects, diffusion processes, radiation effects, for the description of the liquid phase properties (for example, the maximum value of the structural factor, determined by the packing parameter) etc. then the importance of the development of the pseudopotential theory for the metals for which it is valid appears indisputable. An important advantage of this approach is also its simplicity enabling many complicated problems to be discussed not only at the qualitative but also at the quantitative level, and the close relationship with the chemical bond language used in the material science (Gornostyrev et al 1994). For the clarification of the physical mechanism of the Ir dispersion curve flattening the results of band calculations are given in $\S 2$, on which basis the Kohn singularities in the phonon spectra are discussed. In $\S 3$ a modification of the pseudopotential form permitting these effects to be partially simulated for the purpose of an optimal description of the phonon spectra is proposed. In $\S 4$ the characteristics of the phonon spectra extracted from the experimental data on the Ir and Rh heat capacity are given with a detailed description of the corresponding treatment procedure. $\S 5$ presents the calculation results of the lattice heat capacity, and their comparison with the experiment. $\S 6$ summarizes the results of the paper.

\section{§2. Fermi surface and Kohn Singularities in Ir.}

Generally, one could think that the high accuracy of the description of elastic moduli, and hence, of phonon spectra at small wave vectors $q$, provide a good agreement of the theory with experiment for the dispersion curves in the larger part of the Brillouin zone. Significant deviations from the "smooth" behavior of the phonon frequencies depending on the wave vector $\mathbf{q}$ can be connected, first of all, with the Kohn singularities (Izymov and Chernoplekov 1995) when $q$ coincides with the Fermi surface diameter $2 k_{F}$ in the corresponding direction. However in the three dimensional case the typical Kohn singularities in the phonon frequencies $\omega(\mathbf{q})$ are of the order $\left(q-2 k_{F}\right) \ln \left|q-2 k_{F}\right|$ and are weakly expressed. The Kohn singularities clearly expressed in $\omega(\mathbf{q})$ can be only observed in the case of the Fermi surface flattening in one (cylindrical sections) or two (flat sections) directions when $\delta \omega(\mathbf{q}) \sim \pm \sqrt{\left|q-2 k_{F}\right|} \Theta\left( \pm\left(q-2 k_{F}\right)\right)(\Theta(x>0)=$ 
$1, \Theta(x<0)=0$ ) or $\delta \omega(\mathbf{q}) \sim \ln \left|q-2 k_{F}\right|$, respectively (Katsnelson, Naumov and Trefilov 1994). Figs. 1,2 present the results of our calculations of the Fermi surface of Ir and nonuniform susceptibility

$$
\chi(\mathbf{q})=2 \sum_{n n^{\prime}} \int \frac{d \mathbf{k}}{(2 \pi)^{3}} \frac{f\left(\varepsilon_{n}(\mathbf{k})\right)\left[1-f\left(\varepsilon_{n^{\prime}}(\mathbf{k}+\mathbf{q})\right)\right]}{\varepsilon_{n^{\prime}}(\mathbf{k}+\mathbf{q})-\varepsilon_{n}(\mathbf{k})}
$$

where $\varepsilon_{n}(\mathbf{k})$ is the electron energy spectrum; $n, n^{\prime}$ are the band indices; $f(\varepsilon)$ is the Fermi function; integration over $\mathbf{k}$ goes over the Brillouin zone (calculation method see in review by Katsnelson et al 1994). The function $\chi(\mathbf{q})$ determines the form and magnitude of the Kohn singularities in $\omega(\mathbf{q})$. In fig. 1 the flattened sections of the Fermi surface in the directions $\langle 100\rangle$ and $\langle 110\rangle$ are clearly

seen. Fig. 2 shows the maxima of $\chi(\mathbf{q})$ at the relevant values of wave vectors (in the case of the spherical Fermi surface the maxima should have manifested themselves only in $d \chi / d q)$. At the same time in the calculations of the phonon spectra in terms of the pseudopotential model in the second order of the perturbation theory (Greenberg et al 1990, Ivanov et al 1994) the character of the Kohn singularities corresponds to the assumption about the sphericity of the Fermi surface and, therefore, the phonon spectrum in the relevant region of the q space, naturally, proves to be too rigid. If we want to improve the description of phonon spectra in terms of the simple pseudopotential approach, the softening of the phonon frequencies due to the non-sphericity of the Fermi surface can be simulated and described by means of changing the form of the Fourier image of pseudopotential $V_{p s}(q)$ at $0.6(2 \pi / a) \lesssim q \lesssim(2 \pi / a)$ ( $a$ is the lattice constant), determined from the band calculations results. It is this circumstance that should be primarily taken into account in changing the pseudopotential model for Ir and Rh.

\section{$\S 3$. Calculation of Ir and Rh Phonon spectra}

In the calculation of phonon spectra, elastic moduli, and the interatomic interaction potential in papers by Greenberg et al 1990, Ivanov et al 1994 one of the common pseudopotential models (Heine and Weaire 1970; Browman and Kagan 1974; Vaks and Trefilov 1977) was used. The Fourier component of the local pseudopotential of the electron-ion interaction was taken in the form of the Animalu-Heine pseudopotential

$$
V_{p s}^{A H}=-\frac{4 \pi Z}{q^{2} \Omega_{0}}\left[\cos q r_{0}-V_{0}\left(\frac{\sin q r_{0}}{q r_{0}}-\cos q r_{0}\right)\right] \exp \left[-\xi\left(\frac{q}{2 k_{F 0}}\right)^{4}\right]
$$

where $\Omega_{0}$ is the atomic volume, $Z$ is the effective ion charge, $r_{0}, V_{0}$ are the fitting parameters determined from the condition of the agreement with experiment $\Omega_{0}$ and shear modulus $B_{44}$ at pressure $p=0 ; \xi$ is the constant whose value is selected proceeding from the consideration of fast enough convergence of the sums over the reciprocal lattice vectors (here and below the atomic units are used). The screening function of the electron gas was taken in the approximation 
by Geldart and Taylor 1970. See Greenberg et al 1990, Ivanov et al 1994 for the details of the theoretical model, including the substantiation of the choice of values $Z=4.5$ for Ir and $Z=3.86$ for Rh. As discussed in the introduction this model provides a high enough accuracy of the description of elastic moduli and much lower one in the description of the phonon frequencies near the Brillouin zone boundaries. The latter is the result of neglecting the nonsphericity of the Fermi surface, which gives no possibility to describe adequately the Kohn singularities in the phonon spectra (see $\S 2$ ).

For the purpose of simulating these effects remaining in the framework of the simplest pseudopotential model, we considered various possible changes in the form of the psedopotential form factor

$$
V_{p s}(q)=V_{p s}^{A H}(q)+\delta V_{p s}(q)
$$

at large $q$. It is seen from the results of $\S 2$ that the "dangerous" region of the wave vectors, containing the strongest Kohn singularities (which is the result of the maximum non-sphericity of the Fermi surface in the relevant directions) is approximately $\left(0.5 \leq g_{1} \leq 0.7\right) g_{1}$, where $g_{1}$ is the minimum vector of the reciprocal lattice. The addition to the pseudopotential $\delta V_{p s}(q)$ to simulate these singularities should be noticable in the relevant region of $q$ values and vanishingly small at $q=0$ and $q \geq g_{1}$. It is natural to expect that the addition $\delta V_{p s}(q)$ would not noticably affect the calculation values of pressure and elastic moduli which are solely determined by the behavior of $V_{p s}(q)$ at $q \rightarrow 0$ and by $V_{p s}(\mathbf{g})$ for the reciprocal lattice vectors. The concrete form of the addition $\delta V_{p s}(q)$ is rather arbitrary, however it should affect the $V_{p s}(\mathbf{q})$ in the region of $q$ specified at the end of $\S 2$. The following dependences satisfying the above mentioned condition were actually studied:

$$
\begin{gathered}
\delta_{1} V_{p s}(q)=\beta\left(q r_{0}\right) \exp \left[-\alpha\left(q r_{0}\right)^{4}\right] \\
\delta_{2} V_{p s}(q)=\beta\left(q r_{0}\right)^{2} \exp \left[-\alpha\left(q r_{0}\right)^{4}\right] \\
\delta_{4} V_{p s}(q)=\beta\left(q r_{0}\right)^{2} \exp \left[-\alpha\left(q-q_{0}\right)^{2} r_{0}^{2}\right] \\
\delta_{5} V_{p s}(q)=\beta\left(q r_{0}\right)^{2} \exp \left[-\alpha\left(q-q_{0}\right)^{4} r_{0}^{4}\right]
\end{gathered}
$$

where parameters $\beta, \alpha$ (as well as $r_{0}, V_{0}$ ) are found from conditions $\Omega_{0}=\Omega_{0}^{\exp }$, $\Theta_{L T}=\Theta_{L T}^{e x p}, \omega_{X}=\omega_{m a x}^{e x p}$. However, exact fitting to these conditions turned out to be impossible, so the set of parameters giving the best approximation was used. Quantity $\omega_{X}$ is the frequency of phonons at the $X$ point of the Brillouin zone (this is the point where the phonon spectrum calculated using the Animalu-Heine pseudopetential used as a basis for the fitting procedure reaches its maximum). The experimental values of $\omega_{\max }$ were taken from results of the tunnel experiments by Kraynukov et al 1989 for Ir and Zhalko-Titarenko et al 1989 for Rh. The experimental of $\omega_{\max }$ and calculated values of $\omega_{X}$ are listed if table 2 for Ir and table 3 for Rh. $q_{0}$ is the wave vector at the point $\mathrm{X}$ of the Brillouin zone. Among the additions (4a)-(4d) (4c) gives the best agreement of the calculated phonon spectra with the experiment, although the choice of the 
addition if the form (4a) permits nearly the same agreement between the theory and the experiment to be reached. It is important to emphasize that parameter $\beta$ is choosen so that additions (4) were small without giving significant distorsion of the $V_{p s}(q)$ form when comparing with the standart Animalu-Heine model. Nevertheless, calculation results for the phonon spectrum (but not for $q \rightarrow 0$, i. e. not for elastic moduli) proves to be very sensitive to the explicit form of these small additions. The sensitivity of the calculation results to the form of the pseudopotential will be considered in detail in a separate paper. Table 1 lists the parameters for two versions of the pseudepotential, $V_{p s}^{A H}(q)$ and $V_{p s}^{A H}(q)+\delta V_{p s}(q)$, where addition $\delta V_{p s}(q)$ is choosen as (4c) which, comparing with the rest possibilities investigated gives the best description of the lattice properties of Ir and Rh. Tables 2 and 3 and figs. 3,4 give the results of the corresponding calculations. $\Theta_{L T}=\Theta_{D}(T=0)$ is the low temparature value of the Debye temperature calculated from the theoretical values of $B_{i k}$, using as the bulk modulus $B$ its static value $B_{s t}$ determined by differentiating the total energy over volume. Quantity $B_{d y n}$ obtained from the phonon spectra by extrapolation has a methodical meaning: difference $\left|B_{s t}-B_{d y n}\right|$ characterizes the value of multiion interactions (Browman and Kagan 1974). For Ir and Rh its value is substantially smaller than for other transition metals (Vaks et al 1989). Quantity $\gamma_{0}=-\partial \ln \Theta_{L T} / \partial \ln \Omega$ is the low temperature Gruneisen parameter. Note the high sensitivity of $\gamma_{0}$ to the form of the pseudopotential (see tables 23 ). We will discuss the sensitivity of results to the form of the pseudopotential in a separate paper. Unfortunately, at present we don't have experimental results to compare with the calculated values of $\gamma_{0}$. The arrows in figs. 3,4 show the positions of the Kohn singularities in the approximation of free electrons $|\mathbf{q}+\mathbf{g}|=2 k_{F}$. An unusual feature of the considered phonon spectra is that in one of the branches in the $\mathrm{X}-\mathrm{W}$ directions $\omega(\mathbf{q})$ is practically independent on $q$. This feature is not connected with the Kohn singularities in any way and is obtained even in the approximation of the nearest neighbours for interatomic interactions. As discussed in papers by Ivanov et al 1994, Gornostyrev et al 1994 this approximation describes reasonably the elastic moduli of Ir.

It is seen that by introducing "simulation" corrections into $V_{p s}(q)$ one can reach a better description of the phonon spectra of Ir and apparently Rh. This allows us to expect that the calculated phonon spectra can be used for obtaining true enough information on the thermodynamical properties of these metals. It should be emphasized that it is difficult to obtain the information about the phonon spectra of Ir using the method of inelastic neutron scattering because of the large cross section of neutron absorption. Therefore when considering the agreement between the theory and experiment, it is important to get the information on the Ir lattice dynamics from the analysis of the temparature dependences of the thermodynamical properties of Ir, primarily its heat capacity. In the next section we shall use this procedure for obtaining the values of different characteristics (momenta) of the Ir and Rh phonon spectra. 


\section{§4. Analysis of experimental data on IR and Rh heat CaPacity}

The lattice heat capacity is determined by the form of phonon density of states $g(\omega)$. Some characteristics of the latter, which can be used for the comparison of our description of phonon spectra with the experiment, can be derived from the analysis of the temperature dependence of the heat capacity at a constant pressure $C_{p}(T)$.

In this section we identified the lattice component in the heat capacity measured in the experiment and determined values of some average frequencies (momenta) of the phonon spectra.

We used the following set of data in the analysis: in region $12-250 \mathrm{~K}-$ direct experimental data (Clusius and Losa 1959) and in region 1.2-12 $\mathrm{K}$ - the data from the handbook by Hultgren et al 1973, using the data by Wolcott 1956 for the region $1.2-20 \mathrm{~K}$.

In the analysis we neglected the temperature dependence of the coefficient of the electron heat capacity and assumed that the anharmonicity is weak. The assumption on the smallness of the anharmonicity contributions to the thermodynamical properties of metals is justified as it was verified by direct calculations and proved to be true even for such metals with soft phonon modes as alkali ones (Vaks, Kravchuk and Trefilov 1980). With these assumptions the experimentally measured heat capacity at constant pressure $C_{p}$ is described by the relationships

$$
\begin{gathered}
C_{p}=C_{p h}+C_{a} \\
C_{a}=\left\{\gamma+(A-\gamma)\left(C_{p h} / 3 R\right)^{2}\right\} T
\end{gathered}
$$

where $C_{p}$ is the molar heat capacity at constant pressure;

$C_{p h}$ is the phonon component of the heat capacity in the harmonic approximation;

$C_{a}$ - contains the contributions to the heat capacity, having the linear temperature dependence caused by anharmonic effects, thermal expansion of the lattice as well as by the conduction electrons; and here

$\gamma$ is the coefficient of the electron heat capacity at low temperatures;

$A$ is the coefficient at the linear temparature term at high termperatures;

$R$ is the gas constant.

Interpolation formula ( $5 \mathrm{~b}$ ) for $C_{a}$ gives the relevant linear temperature asymptotes both at low and high temperatures and ensures a smooth transition between the low- and high temperature asymptotes by a law similar to the NernstLindeman formula (Reznitskii 1981).

In the high temparature region the phonon component of the heat capacity was described by the expression proposed by Naumov (Naumov 1994):

$$
\begin{gathered}
C_{p h}=3 R\left\{1-\frac{1}{12}\left(\frac{\Omega_{2}}{T}\right)^{2}+\frac{1}{240}\left(\frac{\Omega_{4}}{T}\right)^{4}+\varphi\left(\frac{\Omega_{*}}{T}\right)\right\} \\
\varphi(z)=\frac{z^{2} \exp (z)}{(1-\exp (z))^{2}}-\left(1-\frac{1}{12} z^{2}+\frac{1}{240} z^{4}\right)
\end{gathered}
$$


Here the asymptotic expansion of the phonon heat capacity by small parameter $z=\Omega / T$ is used:

$$
C_{p h}=3 R\left(1-\sum_{n=2}^{\infty} \frac{(n-1) B_{n}}{n !}\left(\frac{\Omega_{n}}{T}\right)^{n}\right)
$$

where $B_{n}$ are the Bernulli numbers $\left(B_{2}=1 / 6, B_{4}=-1 / 30, B_{6}=1 / 42\right.$, $B_{8}=-1 / 30, B_{10}=5 / 66$ and for all odd $n$ all $B_{n}=0$ beginning from $n=3$ ).

In (6) corrections of second and fourth order by $\Omega / T$ are written separetaly, and function $\varphi\left(\frac{\Omega_{*}}{T}\right)$ allows for all higher order corrections in the "Einstein" approximation, i. e. setting $\Omega_{n}=\Omega_{*}$ for all $n \geq 6$.

Quantities $\Omega_{n}$ characterize the momenta (average frequencies) of the phonon spectrum according to the relationship

$$
\left(\Omega_{n}\right)^{n}=<\omega^{n}>=\int_{0}^{\infty} g(\omega) \omega^{n} d \omega / \int_{0}^{\infty} g(\omega) d \omega,
$$

$g(\omega)$ being the phonon density of states.

Quantity $\gamma$ in $C_{a}$, (5b) was determined by the standart procedure: by the approximation of the heat capacity in the low temparature region (particulary $1.2-15 \mathrm{~K}$ ) by a relationship such as $C_{p}=\gamma T+\beta T^{3}+\alpha T^{5}$. Quantities $\gamma, \beta$ and $\alpha$ as well as the value of the Debye temperature in the low temperature limit $\Theta_{L T}$, related to $\beta$ by the equality $\beta=12 \pi^{4} R /\left(5 \Theta_{L T}^{3}\right)$ are listed in the table 4 . The estimates of $\gamma$ and $\Theta_{L T}$ obtained from the set of all used data (Clusius and Losa 1959, Wolcott 1956) agree resonably well with the data available from the literature (see table 4 ).

As for quantities $A, \Omega_{2}, \Omega_{4}$ and $\Omega_{*}$ they were determined by the method of least squares, approximating the heat capacity by relations $(6,7)$ in the temperature region 50-250 K. Within this temperature range these relationships described the experimental results of Clusius and Losa 1959 with a mean-square deviation of about $0.6 \%$. The values of parameters $A, \Omega_{2}, \Omega_{4}$ and $\Omega_{*}$, determined by the least square method are listed in table 4 . The table also gives the value of the Debye temperature in high temperature limit $\Theta_{H T}=\Theta_{D}(T)$, where $\Theta_{L T} \leq T<T_{m}$ ( $T_{m}$ is the melting temperature) related to the second momentum of the phonon spectrum by: $\Omega_{2}=\Theta_{H T} \sqrt{3 / 5}$ (Maradudin, Montroll and Weiss 1963).

The described analisys permitted phonon contribution $C_{p h}$ in the harmonic approximation to be separated from the total heat capacity in the temperature region below $273 \mathrm{~K}$, excluding the electron and anharmonic contributions. Some momenta of the phonon spectra are expressed directly by the integrals of the phonon heat capacity (Junod 1980).

$$
\begin{aligned}
& <\omega>=2 \int_{0}^{\infty}\left(1-\frac{C_{p h}}{3 R}\right) d T \\
& <\omega^{-1}>=\frac{3}{\pi^{2}} \int_{0}^{\infty} \frac{C_{p h}}{3 R} T^{-2} d T
\end{aligned}
$$




$$
\begin{gathered}
<\omega^{-2}>=0.138651 \int_{0}^{\infty} \frac{C_{p h}}{3 R} T^{-3} d T \\
<\omega^{-1} \log \omega>=\frac{3}{\pi^{2}} \int_{0}^{\infty} \frac{C_{p h}}{3 R} \log \left(\frac{T}{0.70702}\right) T^{-2} d T
\end{gathered}
$$

We have calculated these momenta. In the region 15-273 K the integration was carried out by the experimental points (Clusius and Losa 1959) while outside this region we extrapolated the heat capacities by a low temperature asymptote $C_{p h}=\beta T^{3}+\alpha T^{5}$ and high temperature asymptote in the model of the Debye spectrum as in Mirmelstein et al 1984. The average frequencies corresponding to this momenta are listed in table 4 . The meaning of the quantities $\Omega_{-2}, \Omega_{-1}$, $\Omega_{1}$ corresponds to the meaning of $\Omega_{n}$ in relationship (9) for $n=-2,-1$ and 1 , while the meaning of $\Omega_{l o g}$ is determined by

$$
\log \left(\Omega_{\log }\right)=\frac{<\omega^{-1} \log \omega>}{<\omega^{-1}>}=\int_{0}^{\infty} \frac{g(\omega) \log \omega}{\omega} d \omega / \int_{0}^{\infty} \frac{g(\omega)}{\omega} d \omega
$$

\section{§5. Peculiarities of the temperature dependence of heat Capacity OF IR AND RH}

Although the directly measured quantity is heat capacity, it is more convinient to discuss its temperature dependence via the temperature dependence of the effective Debye temperature $\Theta(T)$. This is related to the circumstance that the $C_{p h}(T)$ reaches the asymptotic $3 R$ rather quickly with the increase of $T$ (and this result will be reproduced in any, even apparently incorrect model of the phonon spectrum, and the dependence $\Theta(T)$ is a finer characteristic determining, in particular, the rate at which $C_{p h}(T)$ reaches the asymptotic (Maradudin, et al 1963).

An unusual feature of Ir and Rh, comparing with the other FCC metals, is the sharp fall of the effective Debye temperature with temperature $\left(\Theta_{L T} / \Theta_{H T} \gtrsim 1.5\right)$. For example according to handbook by Zinoviev 1989 for Ca $\Theta_{L T} \approx \Theta_{H T} \approx$ $230 \mathrm{~K}$, for $\mathrm{Cu} \Theta_{L T} \approx 342 \mathrm{~K}, \Theta_{H T} \approx 310 \mathrm{~K}$, for $\mathrm{Pd} \Theta_{L T} \approx 283 \mathrm{~K}, \Theta_{H T} \approx$ $275 K$. Only in Ni ratio $\Theta_{L T} / \Theta_{H T} \approx 476 K / 345 K \approx 1.4$ differs strongly enough from unity (note, by the way, that in this handbook Ir has a very underestimated value of $\left.\Theta_{H T}\right)$.

For comparing the experimental data on $C_{p h}(T)$ in Ir and Rh the calculations were made using phonon spectra $\omega_{\mathbf{q} \nu}$ that we had obtained

$$
C_{p h}(T)=R \sum_{\mathbf{q} \nu}\left(\frac{\hbar \omega_{\mathbf{q} \nu}}{T}\right)^{2} \frac{\exp \left(\hbar \omega_{\mathbf{q} \nu} / T\right)}{\left[\exp \left(\hbar \omega_{\mathbf{q} \nu} / T\right)-1\right]^{2}}
$$

where the summation over the wave vector is made over the Brillouin zone and $\nu$ is the number of the branch. The effective Debye temparature was derived from the standart equation (Maradudin, et al 1963).

$$
C_{p h}(T)=9 R\left[\frac{T}{\Theta(T)}\right]^{3} \int_{0}^{\Theta(T) / T} \frac{d x x^{4} e^{x}}{\left(e^{x}-1\right)^{2}}
$$


The calculation results are shown in figs. 5,6 and in table 4. It should be noted that the corrected pseudopotential with the addition of the form (4c) describes average frequencies $\Omega_{n}$ much better than the Animalu-Heine pseudopotential proposed by Ivanov et al 1994. For example, the agreement between the theoretical and experimental values of $\Omega_{1}$ for Ir improved from $17 \%$ to $9 \%$ and similarly for the rest $\Omega_{n}$. It should be emphasized that in the calculation of $C_{V}(T)$ the change in the volume with temperature was not taken into account. It may be assumed that taking into account this dependence could improve the agreement between the theory and experiment by some more percent. The difference between the experimental and theoretical values of effective Debye temperature $\left(\left|\left(\Theta_{\text {exp }}-\Theta_{\text {theor }}\right) / \Theta_{\text {theor }}\right|\right)$ does not exceed $10 \%$. It seems surprising that a simple pseudopotential model can provide the description of the lattice heat capacity of transition metals (Ir and Rh) at an accuracy level comparable with such for alkali metals. The fact is really unusual as it is commonly believed that the theory of pseudopotential in its simplest form used in this paper is a priori not applicable to transition metals and has not so high accuracy even for polyvalent $s-p$ metals (e. g. Al). Fig 7 shows the corresponding data on $\Theta_{D}(T)$ for $\mathrm{Na}$ obtained from the calculation results (Vaks et al 1978). The accuracy of the description of the phonon spectra in the pseudopotential model for other transition metals is incomparably lower while, say, for $\mathrm{Ni}$ this model is totally unsuitable (Vals et al 1989).

When phonon spectrum $\omega_{\mathbf{q} \nu}$ is known in the whole Brillouin zone one can calculate the temperature dependence of the average square of the atomic displacements in the harmonic approximation

$$
\overline{x^{2}(T)}=\sum_{\mathbf{q} \nu} \frac{\hbar}{2 M \omega_{\mathbf{q} \nu}} \operatorname{cotanh}\left(\frac{\hbar \omega_{\mathbf{q} \nu}}{2 T}\right)
$$

where $M$ is the atom mass. The corresponding results are shown in fig. 8. As far as we know the experimental data on $\overline{x^{2}(T)}$ in Ir and Rh are currently not available.

\section{$\S 6$. Conclusion}

In conclusion we shall underline the most important results obtained in the paper. The most surprising and interesting finding appears to be that the simplest physical pseudopotential model, we proposed earlier, with appropriate modifications seems to provide a high accuracy of the description of phonon spectra and lattice heat capacity of transition metals Ir and Rh and the accuracy of description is comparable to that for alkali metals. This confirms our earlier consideration (Greenberg et al 1990, Gornostyrev et al 1994) about the applicability of the model of pair interactions for the description of the lattice properties of Ir and Rh. However the effects of non-sphericity of the Fermi surface, substantial for all transition metals cannot be completely accounted for. Nevertheless in Ir (and, as appears in Rh) the role of these effects is reduced to some distorsions of the despersion curves in directions in the Brillouin zone 
$(\langle 100\rangle$ and $\langle 110\rangle)$ and appears to be insignificant in the integral characteristics, i. e. in the heat capacity. This situation would be absolutely impossible if in these metals the Fermi level were at the peak of the density of states $E_{F}$, as, say in $\mathrm{Ni}$ or $\mathrm{Pd}$, because this peak affects substantially the elastic moduli and phonon frequencies in the noticable part of the Brillouin zone (Katsnelson et al 1994). The band calculations show, however (Greenberg et al 1990) that not only peak of $N(E)$ does not exist but also there are no relatively weak van Hove singularities near $E_{F}$ in Ir. Of course in itself it cannot be taken as a direct evidence of the applicability of the psudopotential model (in W, say, $N(E)$ near $E_{F}$ is also a smooth function but a reasonable model pseudopotential cannot be constructed). However this can be considered as a some indication to the possibility of such simple description. In the whole, to solve the question of its applicability a wide range of the lattice properties (thermal expansion, equation of state at finite temperatures, other anharmonic effects, etc.) has to be studied. We shall consider these questions in separate papers.

The study described in this paper became possible partially due to the financial support of the International Science Foundation (Grant RGQ300) and Russian Fund for Fundamental Research (Grant 95-02-06426). 


\section{REFERENCES}

Browman, E. G., And Kagan, Yu. M., 1974, Uspekhi Fiz. Nauk, 112, 369. Budworth, D. W., Hear F. E., and Preston, J., 1960, Proc. Roy. Soc. London, 257, 250.

Clusius, K., And Loza C. G., 1959, Z. Naturforsch., 14A, 23.

Geballe, T. H., Matthias, B. T., Clogston, A. M., Williams, H. J., Sherwood, R. C., and Maita, J. P., 1966, J. Appl. Phys., 37, 1181.

Geldart, D. J. W., And Taylor, R., 1970, Canad. J. Phys., 48, 155.

Gornostyrev, Yu. N., Katsnelson, M. I., Mikhin, A. G., Osetskit, Yu. N., ANd Trefilov, A. V., 1994, Fizika metall. Metallovede., 77(2), 79.

Greenberg, B. A., Katsnelson, M. I., Koreshikov, V. G., Osetskit, Yu. N., Peschanskikh, G. V., Trefilov, A. V., Shamanaev, Yu. F., And Yakovenkova L. I. , 1990, Phys. Stat. Sol. (b), 158, 441.

Heine, V., And Weare, D., 1970, Solid State Physics ( Acad. Press, N. Y.), 24 .

Hultgren, R., Decai, R. D., Hawkins, D. T., Gleiser, M., Kelley, K. K., And Wagman, D. D., 1973, Selected values of the thermodynamic properties of elements (Metals Park, Ohio: Amer. Soc. for Metals), .

Ivanov, A. S., Katsnelson, M. I., Mikhin, A. G., Osetskit, Yu. N., Rumyantsev, A. Yu., Trefilov, A. V., Shamanaev, and Yu. F., Yakovenkova, L. I., 1994, Phil. Mag., B69, 1183.

Izymov, Yu. A., And Chernoplekov, N. A., 1995, Neutron spectroscopy, (Plenum Press, N. Y.).

Junod, A., 1980, Solid State Communications, 33, 55.

Katsnelson, M. I., Naumov, I. I., And Trefilov, A. V., 1994, Phase Transitions, B49, 143.

Kraynukov, S. N., Khotkevich, A. N., Yanson, I. K., Zhalko-Titarenko, A. V., Antonov, V. N., Nemoshkalenko, V. V., Milman, V. Yu., Khlopkin, M. N., AND Shitikov, Yu. L., 1989, Fizika Tverdogo Tela, 31, iss. 3, 123 .

Maksimov, E. G., and Savrasov, S. Yu., 1995, Uspekhi Fiz. Nauk, 165, 773.

Maradudin, A. A., Montroll, E. W., And Weiss, G. H., 1963, Theory of lattice dynamics in the harmonic approximation, (Acad. Pres. N. Y.).

Mirmelstein, A. V., Karkin, A. E., Arkhipov, V. E., And Voronin, V. I., 1984, Fizika Metallov Metallovede, 58, 1008.

Naumov, V. N., 1994, Phys. Rev. B, 49, 13247.

Nemoshkalenko, V. V., Milman, V. Yu., Zhalko-Titarenko, Antonov, V. N., And Shitikov, Yu. L., 1988, Pis'ma ZhETF, 47, 245.

Reznitski, L. A., 1981, Calorimetry of Solids (Moscow, MSU, in Russian), p184.

Vaks, V. G., Kapinos, V. G., Osetskit, Yu. N., Samoliuk, G. D., And Trefilov, A. V., 1989, Fizika Tverd. Tela, 31, 139.

Vaks, V. G., Kravchuk, S. P., and Trefilov, A. V., 1980, J. Phys., F10, 2325. 
Vaks, V. G., And Trefilov, A. V., 1977, Fizika Tverd. Tela, 19, 244.

Vaks, V. G., Zarochentsev, E. V., Kravchuk, S. P., Safronov, V. P., and Trefilov, A. V., 1978, Phys. Stat. Sol. (b), 85, 63.

Wolcott, N. M., 1956, Conference de Physique des Basses Temperatures (Institute de Froid, Paris, 1956), 286.

Zhalko-Titarenko, A. V., Milman, V. Yu., A. V., Antonov, V. N., Nemoshkalenko, Khotkevich, A. V., Kraynukov, 1989, Metallofizika, 11, iss. 5, 25.

Zinoviev, V. E., 1989, Properties of metals at high temperatures, Moscow, Metallurgia, in Russian. 


\section{Captions to tables of the paper by Katsnelson et al "Peculiarities OF PHONON SPECTRA ..."}

Table 1. Pseudopotential parameters (in atomic units) in relations (2), (4). Table 2. Values of energy $E$, elastic moduli $B_{i k}$, their derivatives over pressure, Debye temperature at $T \rightarrow 0 \Theta_{L T}$, Gruneisen parameter $\gamma_{0}$ and maximum phonon frequency $\omega_{\max }$ for Ir. $E-\mathrm{Ry} / \mathrm{at}, \mathrm{P}, \mathrm{B}-10^{11} \mathrm{dyn} / \mathrm{cm}^{2}, \Theta_{L T}-\mathrm{K}$, $\omega_{\max }-\mathrm{THz}$. AH corresponds to the values calaulated by the Animalu-Heine pseudopotential, $4 \mathrm{c}$ - the same with the addition (4c).

Table 3. Values of energy $E$, elastic moduli $B_{i k}$, their derivatives over pressure, Debye temperature at $T \rightarrow 0\left(\Theta_{L T}\right)$, Gruneisen parameter $\gamma_{0}$ and maximum phonon frequency for $\mathrm{Rh} \omega_{\max } . E-\mathrm{Ry} / \mathrm{at}, \mathrm{P}, \mathrm{B}-10^{11} \mathrm{dyn} / \mathrm{cm}^{2}, \Theta_{L T}$ - K, $\omega_{\max }-\mathrm{THz}$.

Table 4a. Parameters characterizing the experimental heat capacity at a constant pressure, low temperature and high temperature limits of the Debye temperature and the phonon spectrum momenta for Ir. AH corresponds to the values calculated by the Animalu-Heine pseudopotential, $4 \mathrm{c}$ - the same with the addition (4c). [Clu] and [Geb] are data sets from Clusius et al 1959 and Geballe et al 1966 respectively. Subcolumn "this paper" in the column "experiment" containes values derived from the experimental data using the technique described in $\S 4$.

Table 4b. Parameters characterizing the experimental heat capacity at a constant pressure, low temperature and high temperature limits of the Debye temperature and the phonon spectrum momenta for Rh. AH corresponds to the values calculated by the Animalu-Heine pseudopotential, $4 \mathrm{c}$ - the same with the addition (4c). [Clu], [Geb] and [Wol] are data sets from Clusius et al 1959, Geballe et al 1966 and Wolcott 1956 respectively. Subcolumn "this paper" in the column "experiment" containes values derived from the experimental data using the technique described in $\S 4$. 
Figure captions to the paper by Katsnelson et al "PeCuliarities of PHONON SPECTRA ..."

Fig.1 Section of the Fermi surface of Ir.

Fig.2 Non-uniform susceptability $\chi(\mathbf{q})$ in directions $\langle 110\rangle$ (a) and $\langle 110\rangle$ (b). Numbers in fig. $2 \mathrm{~b}$ designate contributions from the corresponding branches.

Fig.3 Phonon spectrum in Ir. Dashed line - the phonon spectrum calculated by the Animalu-Heine pseudopotential, solid line - the same with the addition (4c). Experimental points $(\diamond)$ are taken from Ivanov et al 1994. Arrows indicate the Kohn singularities in the approximation of free electrons.

Fig.4 Phonon spectrum in Rh. Dashed line - the phonon spectrum calculated by the Animalu-Heine pseudopotential, solid line - the same with the addition (4c). Arrows indicate the Kohn singularities in the approximation of free electrons.

Fig.5 Dependence of the lattice heat capacity at a constant volume (a) and the effective Debye temperature (b) on temperature for Ir. Dashed line corresponds to the values calculated by the Animalu-Heine pseudopotential, Solid line - the same with the addition (4c). The experimental values derived in this paper denoted as $\diamond . T_{p l}=\hbar \omega_{p l}=\hbar\left(4 \pi Z e^{2} / M \Omega_{0}\right)^{1 / 2}, \omega_{p l}$ is the ionic plasma frequency.

Fig.6 Dependence of the lattice heat capacity at a constant volume (a) and the effective Debye temperature (b) on temperature for Rh. Dashed line corresponds to the values calculated by the Animalu-Heine pseudopotential, Solid line - the same with the addition (4c). The experimental values derived in this paper denoted as $\diamond . T_{p l}=\hbar \omega_{p l}=\hbar\left(4 \pi Z e^{2} / M \Omega_{0}\right)^{1 / 2}, \omega_{p l}$ is the ionic plasma frequency.

Fig.7 Theoretical $(-)$ and experimental $(\diamond)$ temperature dependences of the lattice heat capacity at a constant volume (a) and the effective Debye temperature (b) for Na from Vaks et al 1978. $T_{p l}=\hbar \omega_{p l}=\hbar\left(4 \pi Z e^{2} / M \Omega_{0}\right)^{1 / 2}, \omega_{p l}$ is the ionic plasma frequency.

Fig.8 Average square of atomic displacement in Ir (a) and Rh (b). Dashed line corresponds to the values calculated by the Animalu-Heine pseudopotential, Solid line - the same with the addition (4c). $T_{p l}=\hbar \omega_{p l}=\hbar\left(4 \pi Z e^{2} / M \Omega_{0}\right)^{1 / 2}$, $\omega_{p l}$ is the ionic plasma frequency. 
table 1

\begin{tabular}{|c|c|c|c|c|c|c|c|}
\hline metal & $\Omega_{0}$ & $\mathrm{Z}$ & $\xi$ & $r_{0}$ & $V_{0}$ & $\alpha$ & $\beta$ \\
\hline Ir AH & 95.88 & 4.5 & 0.3 & 2.696 & -1.243 & & \\
\hline Ir 4c & 95.88 & 4.5 & 0.3 & 2.696 & -1.243 & 1.285 & $-4.29 \cdot 10^{-3}$ \\
\hline Rh AH & 92.57 & 3.86 & 0.3 & 2.678 & -1.251 & & \\
\hline Rh 4c & 92.57 & 3.86 & 0.3 & 2.678 & -1.251 & 1.276 & $-6.76 \cdot 10^{-3}$ \\
\hline
\end{tabular}

table 2

\begin{tabular}{|c|c|c|c|c|c|c|c|c|c|c|c|c|c|}
\hline & $\mathrm{E}$ & $\mathrm{P}$ & $B_{s t}$ & $B_{\text {dyn }}$ & $B_{33}$ & $B_{44}$ & $\frac{\partial B_{33}}{\partial P}$ & $\frac{\partial B_{44}}{\partial P}$ & $\frac{\partial B_{s t}}{\partial P}$ & $\frac{\partial B_{\text {dyn }}}{\partial P}$ & $\Theta_{D}$ & $\gamma_{0}$ & $\omega_{\max (X)}$ \\
\hline $\mathrm{AH}$ & -9.064 & 0.00 & 31.27 & 35.17 & 14.01 & 26.69 & 2.50 & 4.63 & 5.95 & 5.90 & 409 & 1.73 & 8.06 \\
\hline $4 \mathrm{c}$ & -9.060 & 0.00 & 29.26 & 33.14 & 14.40 & 24.43 & 2.21 & 5.77 & 6.71 & 6.60 & 401 & 1.99 & 6.98 \\
\hline exper & - & 0.00 & 37.1 & 37.1 & 17.1 & 26.9 & - & - & - & - & 425 & - & 6.91 \\
\hline
\end{tabular}

table 3

\begin{tabular}{|c|c|c|c|c|c|c|c|c|c|c|c|c|c|}
\hline & $\mathrm{E}$ & $\mathrm{P}$ & $B_{s t}$ & $B_{d y n}$ & $B_{33}$ & $B_{44}$ & $\frac{\partial B_{33}}{\partial P}$ & $\frac{\partial B_{44}}{\partial P}$ & $\frac{\partial B_{s t}}{\partial P}$ & $\frac{\partial B_{d y n}}{\partial P}$ & $\Theta_{D}$ & $\gamma_{0}$ & $\omega_{\max (X)}$ \\
\hline $\mathrm{AH}$ & -6.860 & 0.00 & 25.33 & 29.02 & 10.97 & 22.15 & 2.09 & 4.30 & 5.70 & 5.51 & 500 & 1.54 & 10.03 \\
\hline $4 c$ & -6.857 & 0.00 & 22.30 & 25.91 & 11.49 & 18.76 & 1.60 & 6.40 & 7.21 & 6.87 & 482 & 2.05 & 7.70 \\
\hline exper & - & 0.00 & 26.9 & 26.9 & 11.5 & 19.4 & - & - & - & - & 482 & - & 7.63 \\
\hline
\end{tabular}


table $4 \mathrm{a}$

\begin{tabular}{|l||c|c|c|c|c|}
\hline \multicolumn{1}{|c||}{ parameter } & \multicolumn{3}{c|}{ experiment } & \multicolumn{2}{c|}{ theory, this paper } \\
\cline { 2 - 6 } & this paper & {$[\mathrm{Clu}]$} & {$[\mathrm{Geb}]$} & $\mathrm{AH}$ & $4 \mathrm{c}$ \\
\hline$\gamma, \mathrm{mJ} \mathrm{mol}^{-1} \mathrm{~K}^{-2}$ & 3.26 & 3.18 & 3.20 & & \\
$\beta, \mathrm{mJ} \mathrm{mol}^{-1} \mathrm{~K}^{-4}$ & 0.0228 & 0.0244 & & & \\
$\alpha, \mathrm{mJ} \mathrm{mol}^{-1} \mathrm{~K}^{-6}$ & 0.00005 & & & & \\
$\Theta_{L T}, \mathrm{~K}$ & 440 & 430 & & 409 & 401 \\
$\Theta_{H T}, \mathrm{~K}$ & 290 & 289 & & 355 & 321 \\
$A, \mathrm{~mJ} \mathrm{~mol}-1 \mathrm{~K}^{-2}$ & 4.7 & & & & \\
$\Omega_{l o g}, \mathrm{~K}$ & 192 & & & 227 & 214 \\
$\Omega_{-2}, \mathrm{~K}$ & 188 & & & 222 & 213 \\
$\Omega_{-1}, \mathrm{~K}$ & 202 & & & 240 & 224 \\
$\Omega_{1}, \mathrm{~K}$ & 217 & & & 260 & 241 \\
$\Omega_{2}, \mathrm{~K}$ & 225 & & & 267 & 248 \\
$\Omega_{4}, \mathrm{~K}$ & 236 & & & 276 & 261 \\
$\Omega_{*}, \mathrm{~K}$ & 240 & & & & \\
\hline
\end{tabular}

table $4 b$

\begin{tabular}{|l||c|c|c|c|c|c|}
\hline \multicolumn{1}{|c||}{ parameter } & \multicolumn{3}{|c|}{ experiment } & \multicolumn{2}{c|}{ theory, this paper } \\
\cline { 2 - 7 } & this paper & [Clu $]$ & [Bud $]$ & Wol $]$ & AH & 4c \\
\hline$\gamma, \mathrm{mJ} \mathrm{mol}^{-1} \mathrm{~K}^{-2}$ & 4.95 & 4.18 & 4.65 & 4.90 & & \\
$\beta, \mathrm{mJ} \mathrm{mol}^{-1} \mathrm{~K}^{-4}$ & 0.0195 & 0.0213 & & & & \\
$\alpha, \mathrm{mJ} \mathrm{mol}-1 \mathrm{~K}^{-6}$ & 0.00002 & & & & & \\
$\Theta_{L T}, \mathrm{~K}$ & 505 & 450 & 512 & & 500 & 482 \\
$\Theta_{H T}, \mathrm{~K}$ & 345 & 346 & & & 437 & 362 \\
$A, \mathrm{~mJ} \mathrm{~mol}{ }^{-1} \mathrm{~K}^{-2}$ & 5.5 & & & & & \\
$\Omega_{\text {log }}, \mathrm{K}$ & 227 & & & & 283 & 246 \\
$\Omega_{-2}, \mathrm{~K}$ & 222 & & & & 281 & 245 \\
$\Omega_{-1}, \mathrm{~K}$ & 240 & & & & 299 & 256 \\
$\Omega_{1}, \mathrm{~K}$ & 260 & & & & 328 & 273 \\
$\Omega_{2}, \mathrm{~K}$ & 267 & & & & 339 & 280 \\
$\Omega_{4}, \mathrm{~K}$ & 276 & & & & 359 & 293 \\
$\Omega_{*}, \mathrm{~K}$ & 278 & & & & & \\
\hline
\end{tabular}




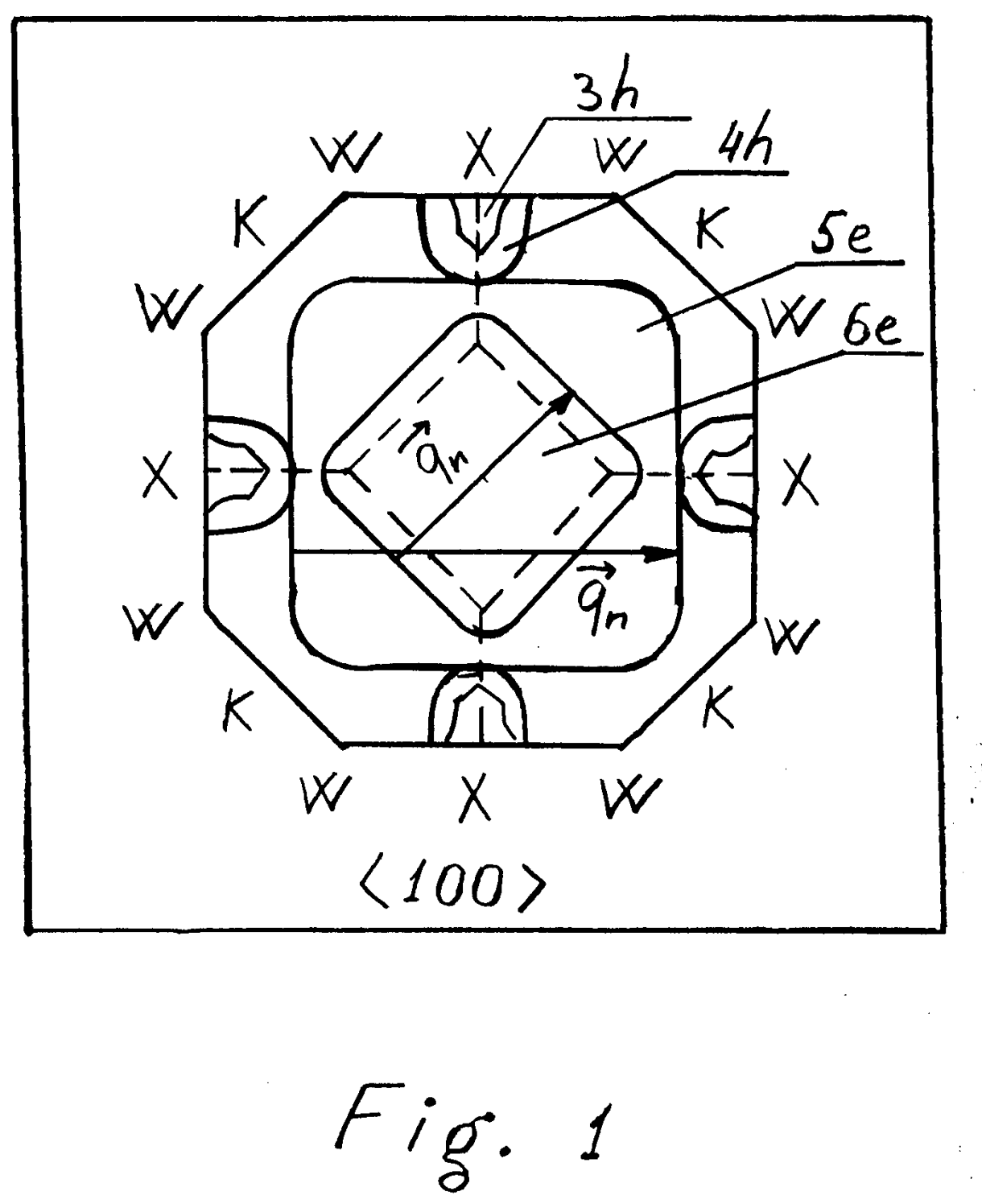



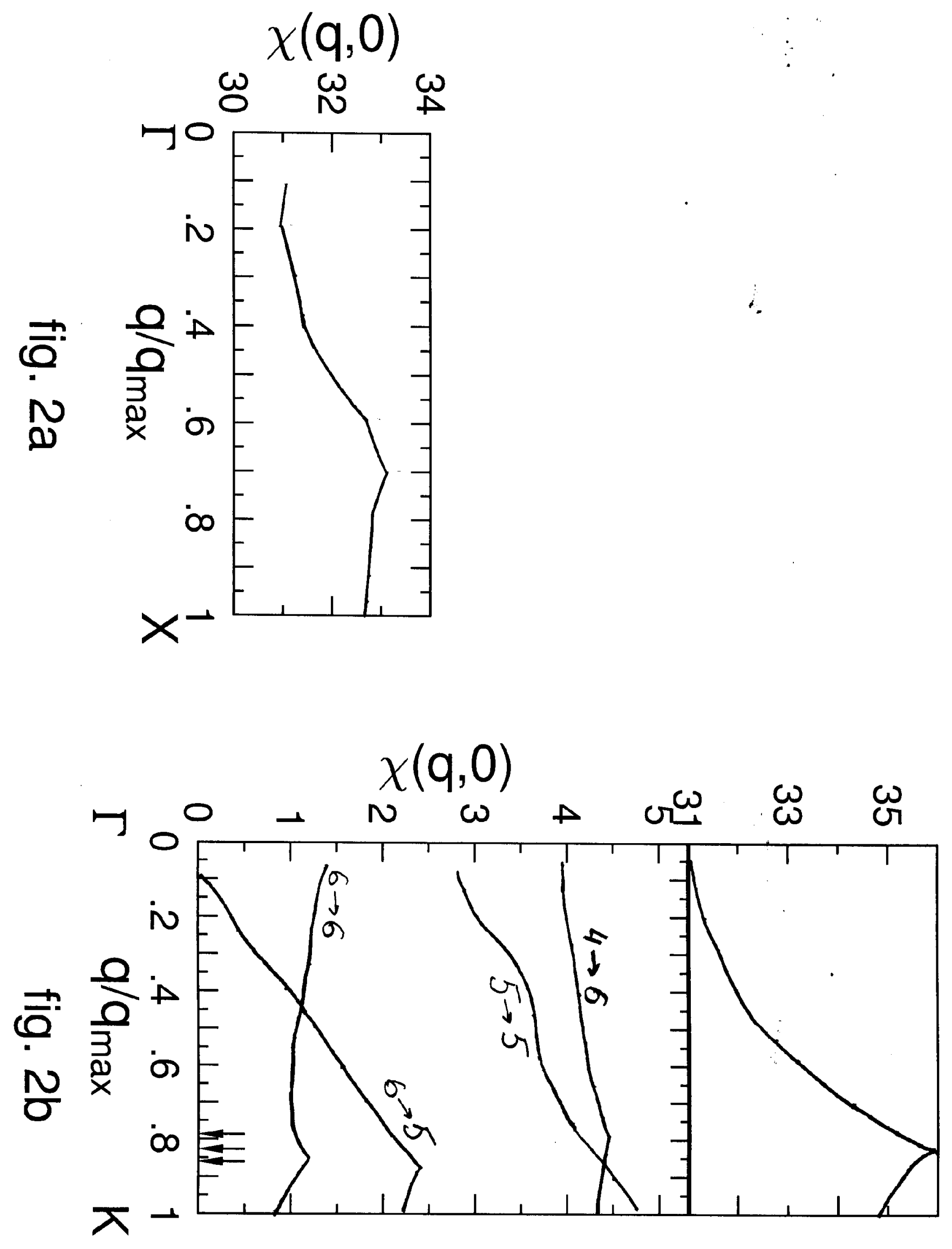


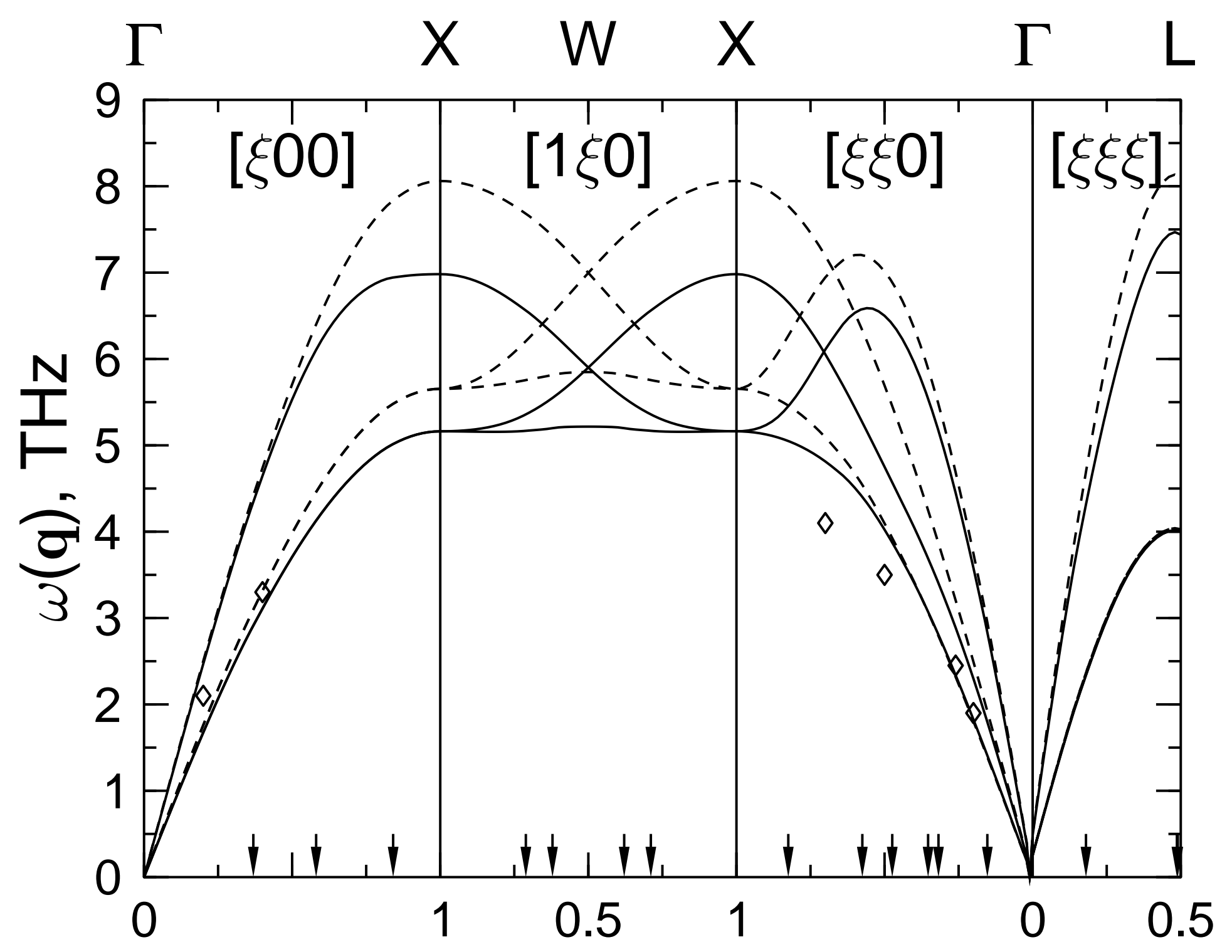

fig. 3 


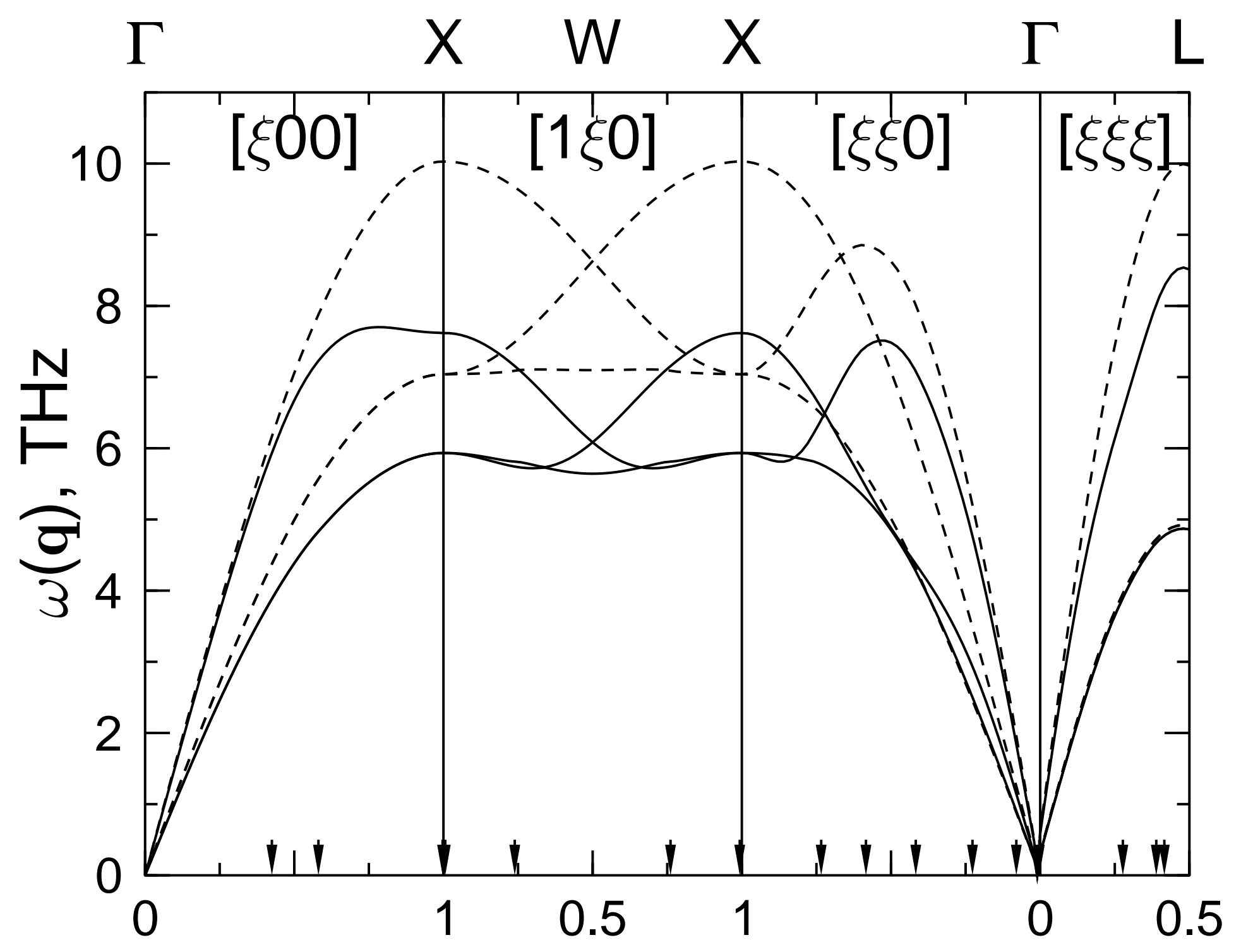

fig. 4 


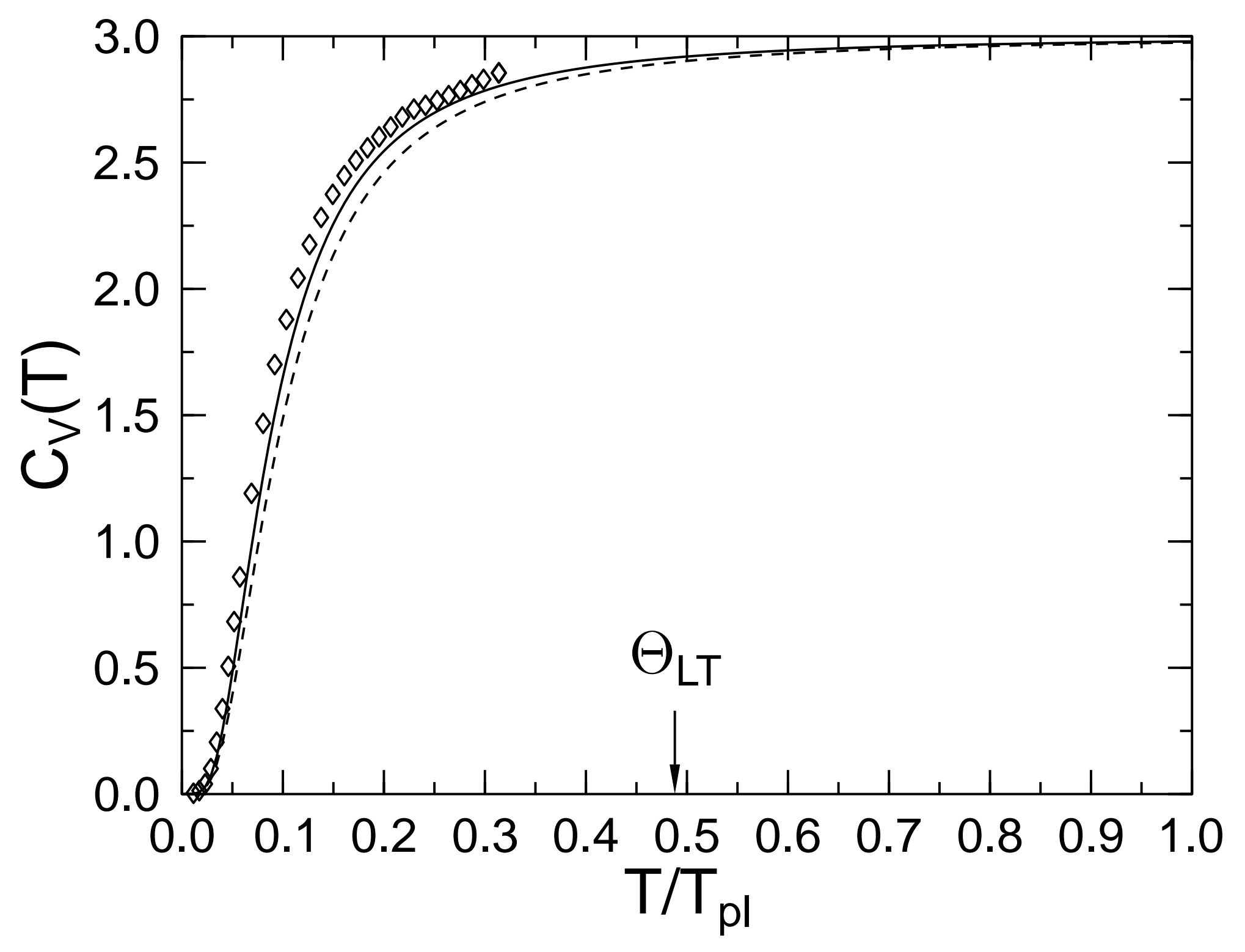

fig. $5 a$ 


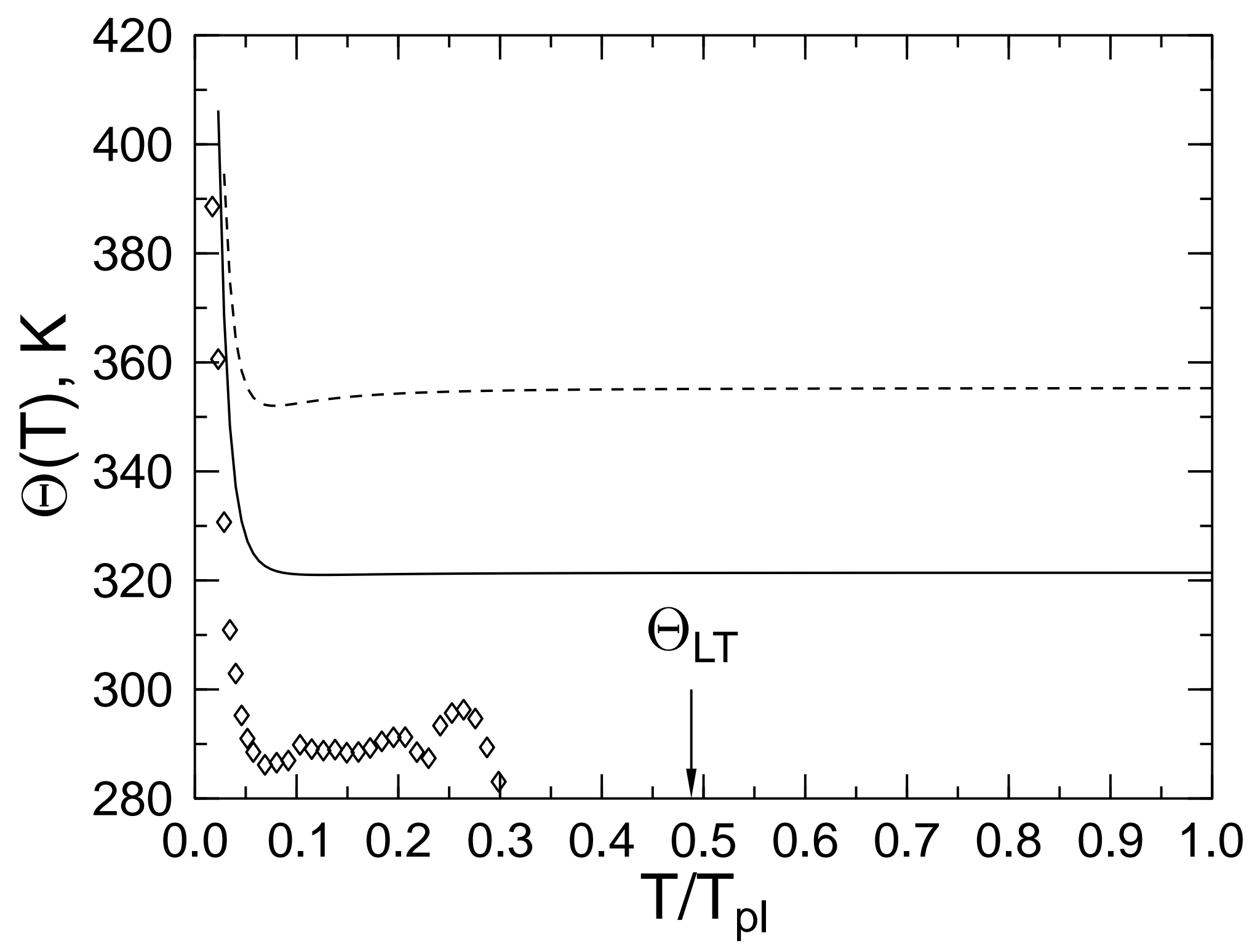

fig. $5 b$ 


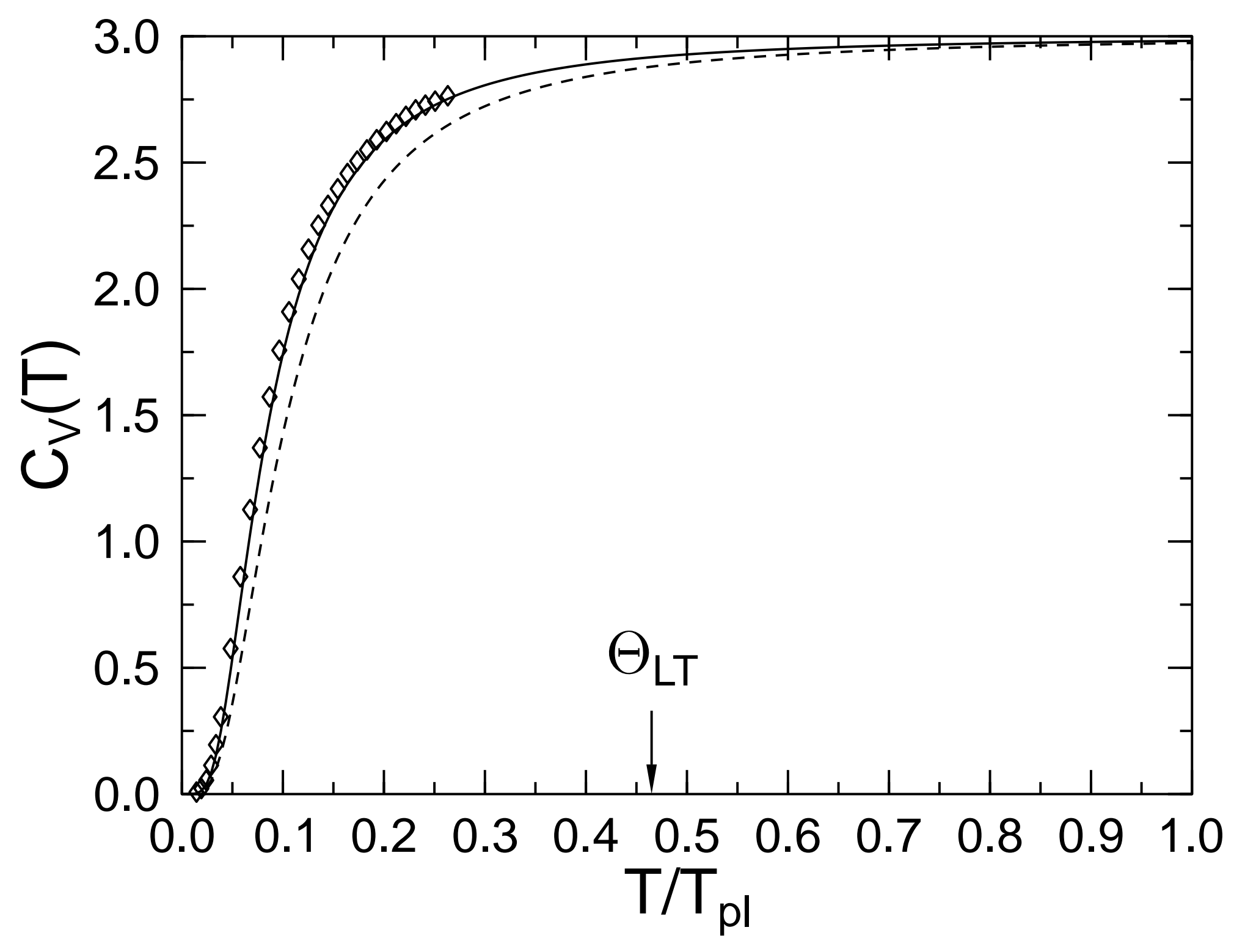

fig. $6 a$ 


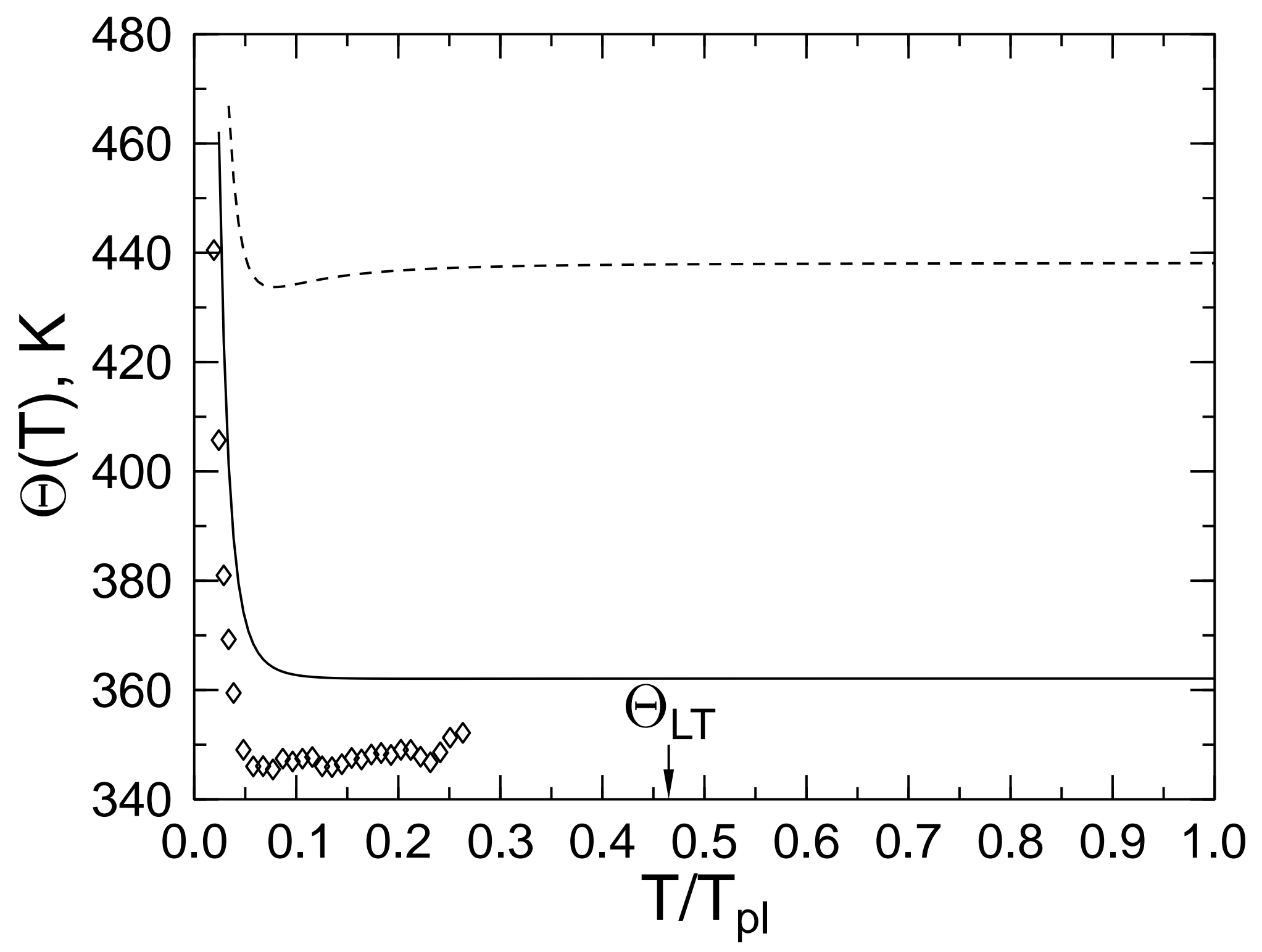

fig. $6 b$ 


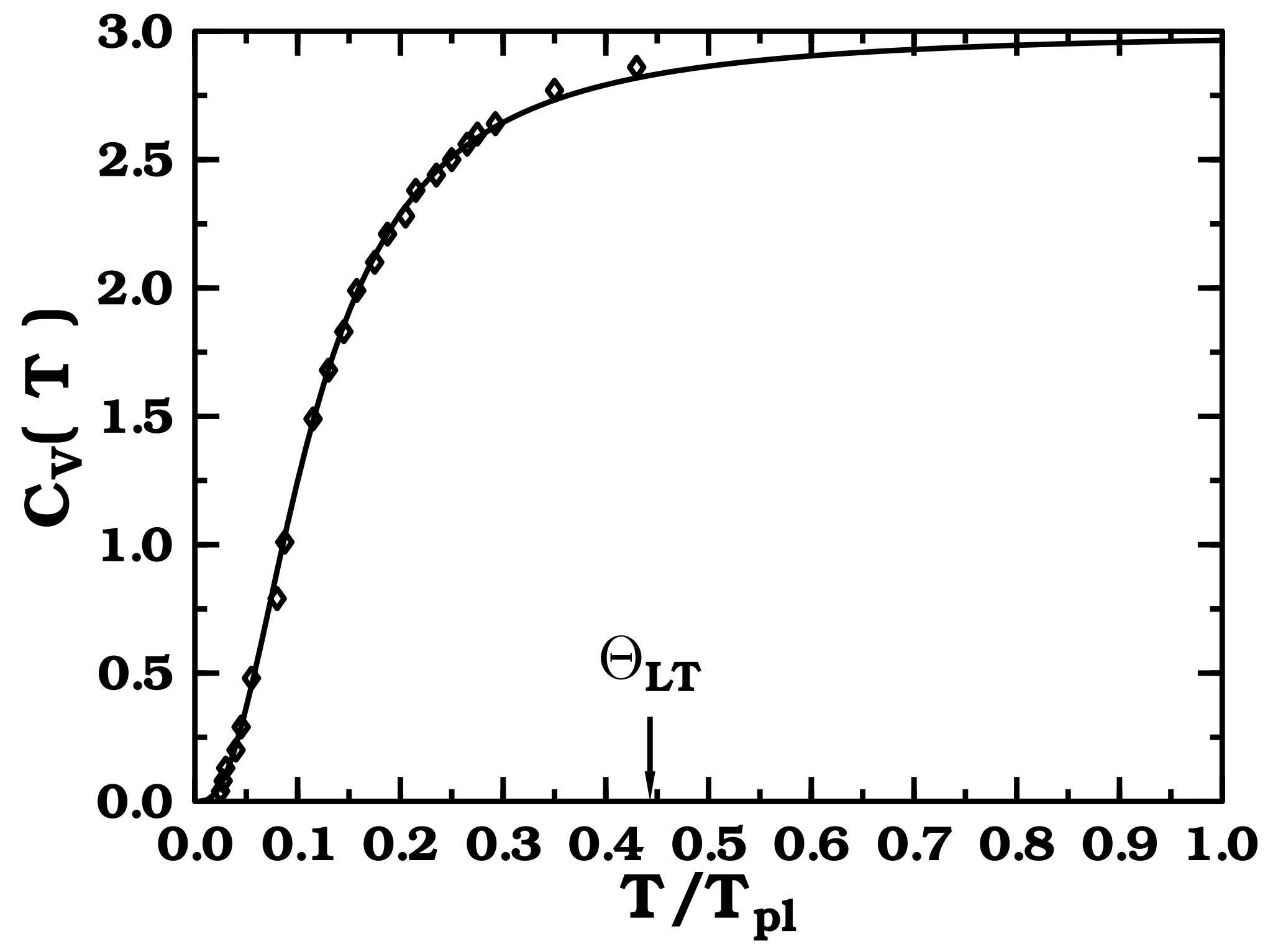

fig.7a - 


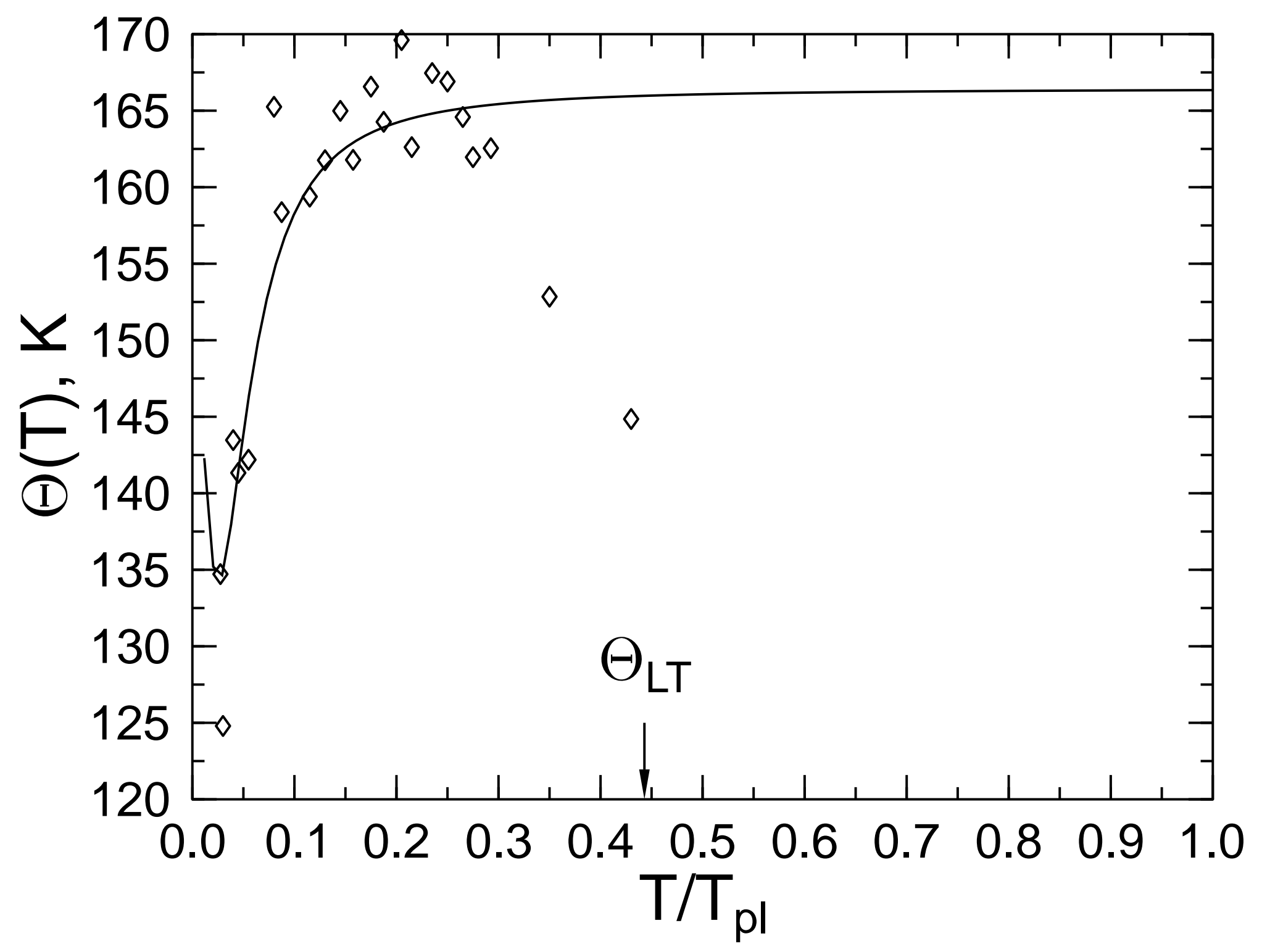

fig. $7 b$ 


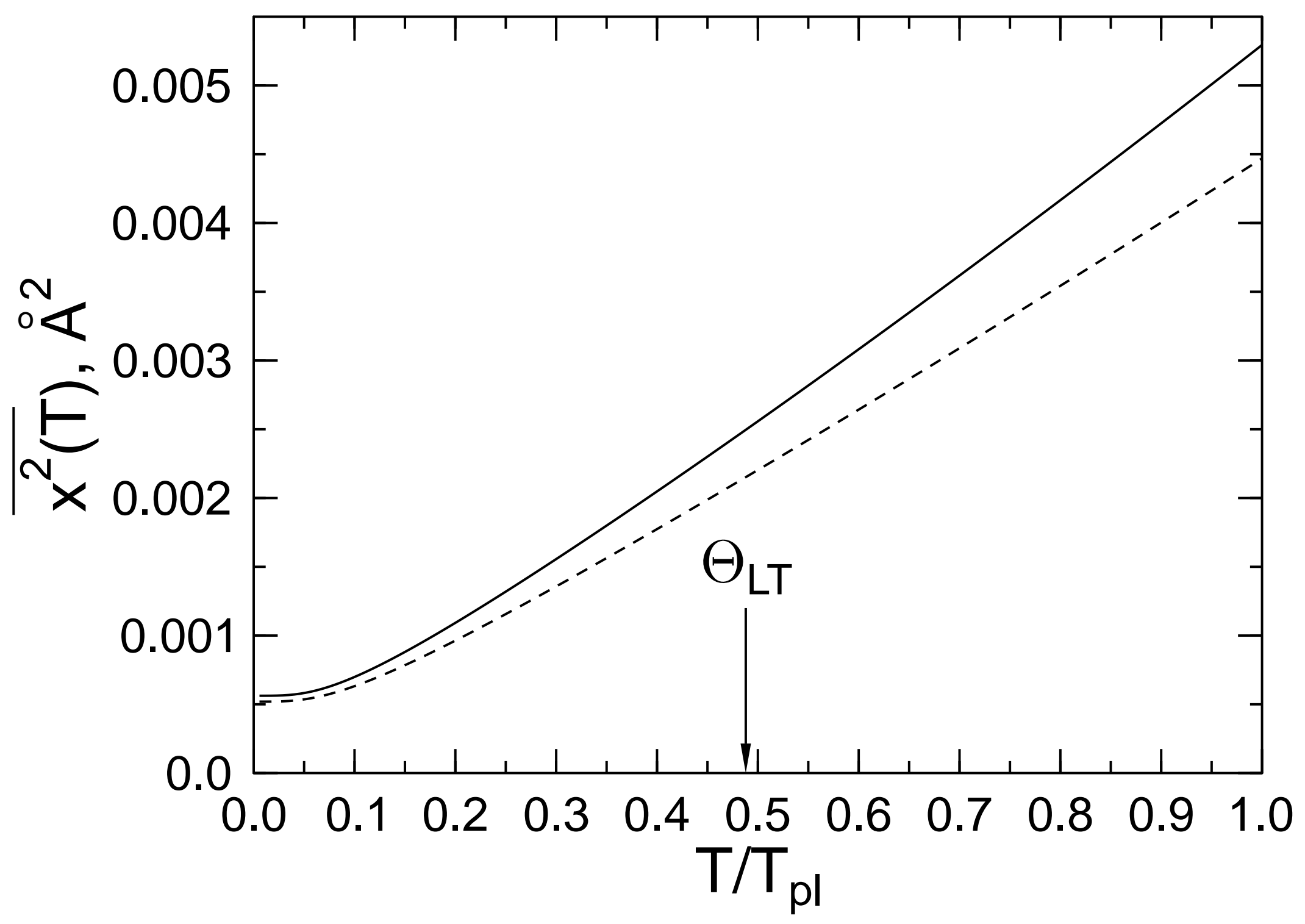

fig. $8 a$ 


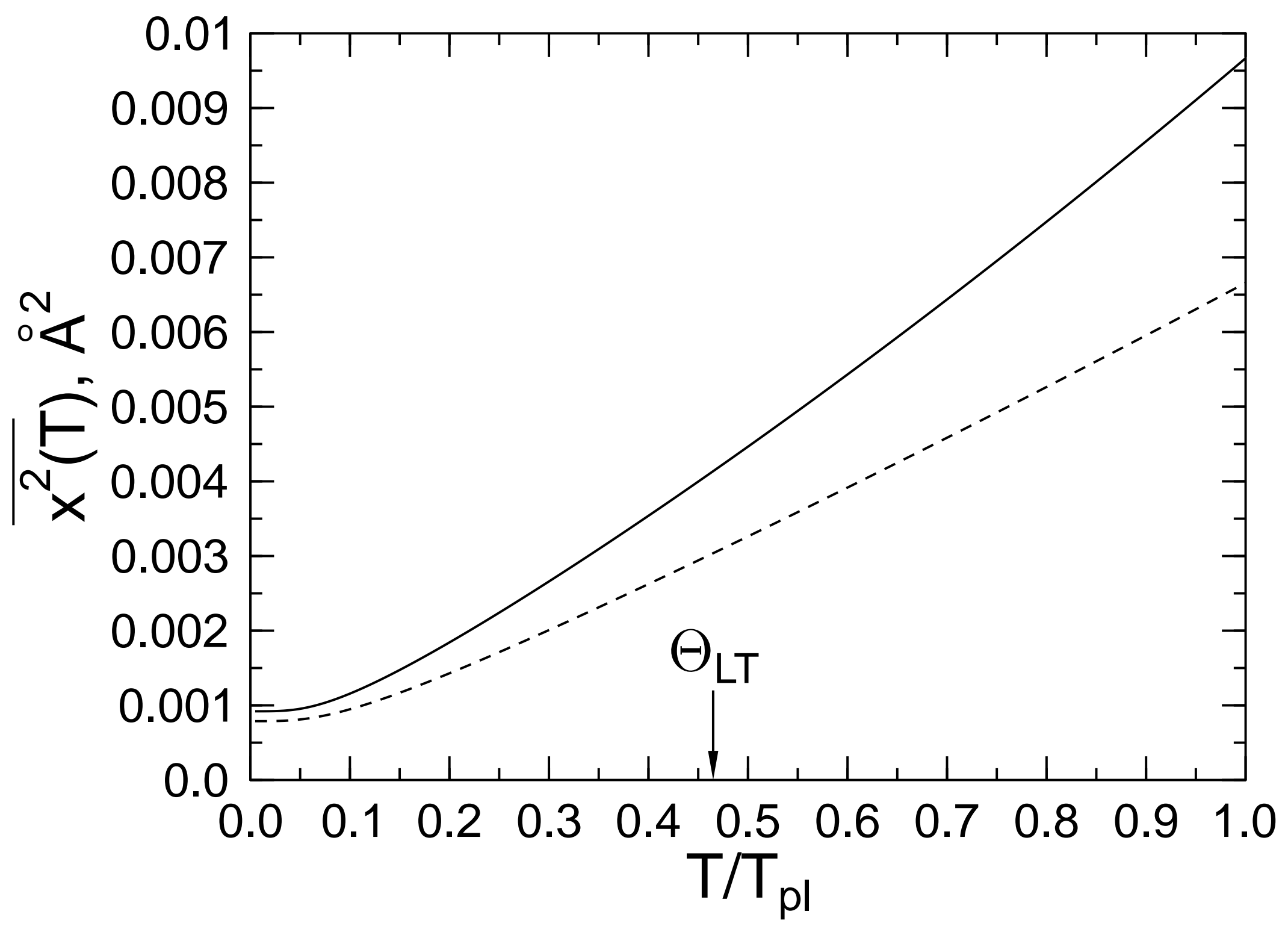

fig. $8 b$ 